\title{
Astronomical activities with disabled people
}

\author{
Amelia Ortiz-Gil ${ }^{1}$, Pere Blay ${ }^{2}$, A. Teresa Gallego Calvente ${ }^{3}$, Miquel \\ Gómez $^{1}$, José Carlos Guirado ${ }^{1}$, Mariana Lanzara ${ }^{1}$ and Silvia \\ Martínez Núñez ${ }^{4}$ \\ ${ }^{1}$ Observatorio Astronómico Universidad de Valencia, \\ Ed. Institutos de Investigación, Pol. La Coma, s/n, 46980 Paterna (Valencia), Spain \\ email: amelia.ortiz@uv.es, miquel.gomez@uv.es, jose.c.guirado@uv.es, \\ mariana.lanzara@uv.es \\ ${ }^{2}$ GACE, Universidad de Valencia
}

Ed. Institutos de Investigación, Pol. La Coma, s/n, 46980 Paterna (Valencia), Spain email: pere.blay@uv.es

${ }^{3}$ Instituto de Astrofísica de Andalucía

Camino Bajo de Huétor, 50. 18008 Granada, Spain email: gallego@iaa.es

${ }^{4}$ Escuela Politécnica Superior, Universidad de Alicante

P.O. Box 9903080 Alicante, Spain email: Silvia.Martinez@ua.es

\begin{abstract}
As we celebrate the International Year of Astronomy, we have been working on four different projects with the goal of making astronomy more accessible to people with special needs. These projects are 1) an astronomy book and web site for blind people, 2) an open source software for people with motor disabilities, 3) a planetarium program for the visually impaired and 4) educational material for intellectually disabled people.
\end{abstract}

Keywords. Public outreach, education, disabled.

\section{Touch the sky web site and book for the blind}

The Astronomical Observatory of Padova started back in 2000 a web site especially designed for the visually impaired. It is built in a way that it can be read by the voice processor of a computer. The drawings have been designed in a way that it optimally translates the original pictures into new ones to be printed in braille.

As we celebrate the International Year of Astronomy, the web site has been updated and is being translated into different languages. One can currently find the English, Italian and Spanish versions. The web site address is http://www.touchthesky .eu

A book has been printed in braille, from the Spanish version, to make the contents more easily reachable to people with no computer or braille printer (Fig. 1).

\section{Open source software for people with motor disabilities}

Some people have motor problems, like those suffering from cerebral palsy or brain injury. Even speaking can be difficult for these persons.

A way to establish communication with the external world is through the use of a computer with a special software. The currently available codes are not open source and have standard settings, so they cannot be adapted to the special characteristics of each person. 

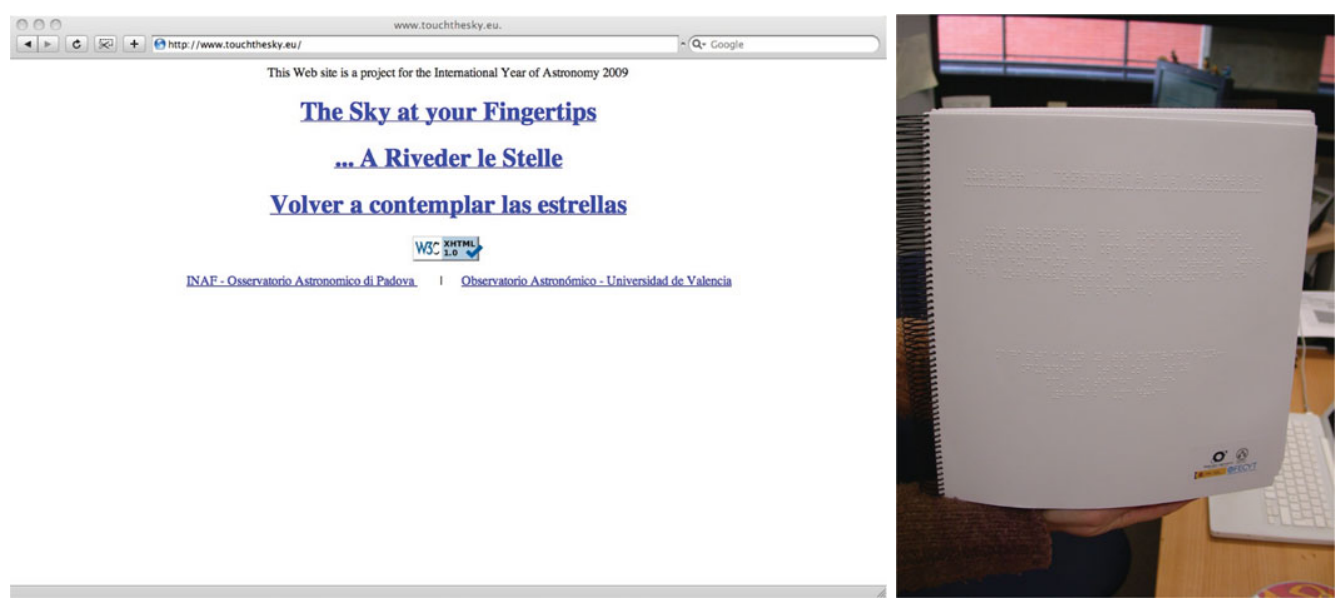

Figure 1. The Touch the sky web site and its book version in Braille.
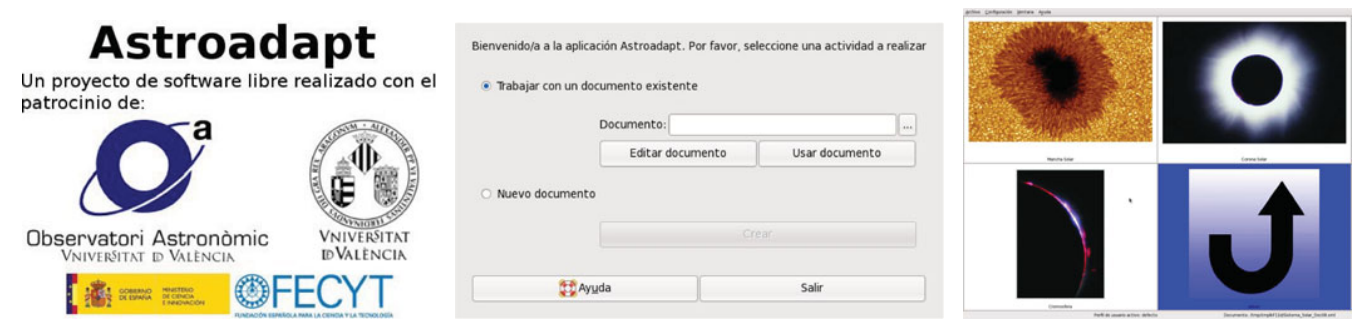

Figure 2. Three screen captures from "Astroadapt".

We have developed an easy-to-adapt open source software far superior to the existent propietary codes. In addition, our software includes astronomical contents in order to make astronomy more accessible to this group of people and it can be easily translated into other languages (Fig. 2).

\section{A planetarium program for the visually impaired}

Following and combining the experiences from previous groups, we have developed a planetarium session for the visually impaired and the blind. The session consists on a walk through different constellations. The recording of the program takes advantage of the sound system of the Hemisferic planetarium at Valencia (Spain). It has seven different speakers distributed over the $24 \mathrm{~m}$-diameter spheric screen that allow us to choose the direction from which the sound is coming. Each constellation has a sound associated to it, as well as some special stars. The public locates the constellation in the sky towards the direction from which they hear the sound.

Two narrators are guiding the tour. One is the normal planetarium narrator. The second guides the public through a tactile sphere (Fig. 3), which has the drawings of the constellations engraved, the stars and the paths to go from one constellation to the next, all of it with patterns raised in different levels.

\section{Educational material for intellectually disabled people}

Following our own experience organising astronomical workshops with intellectually disabled people, we have compiled a number of resources and materials that can be used 


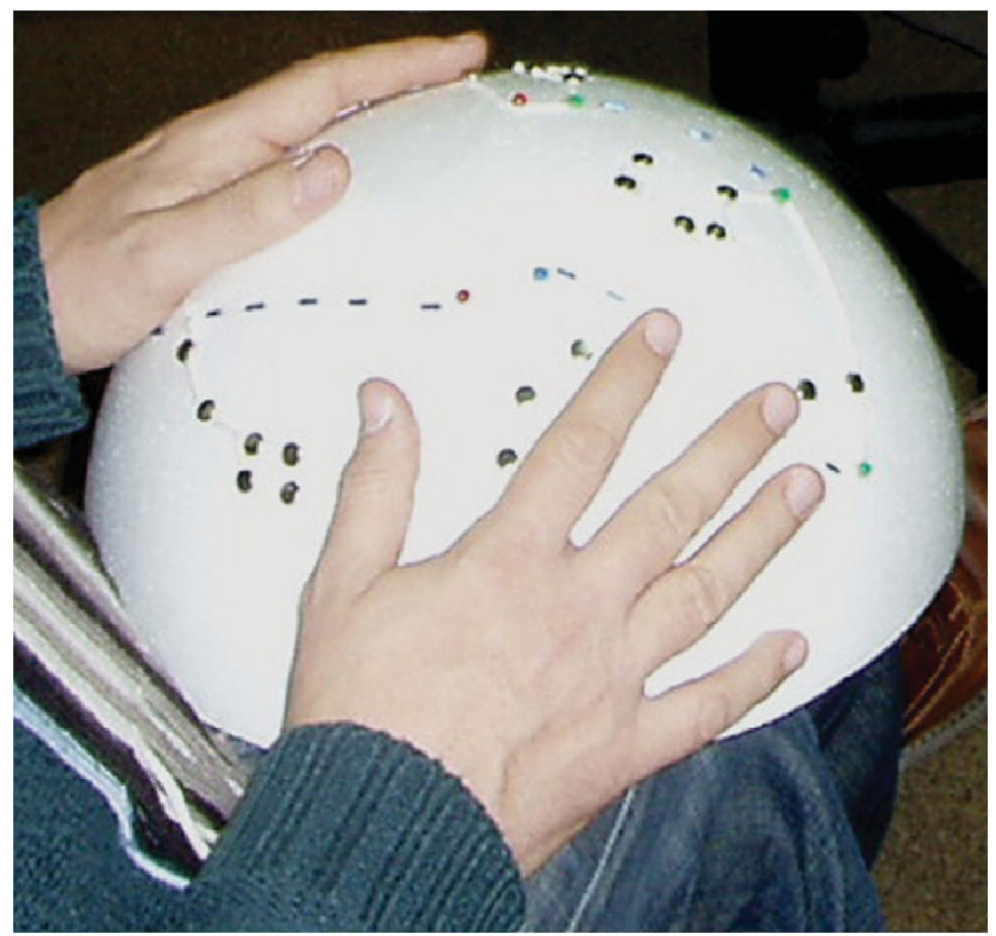

Figure 3. Tactile sphere to follow the planetarium session.

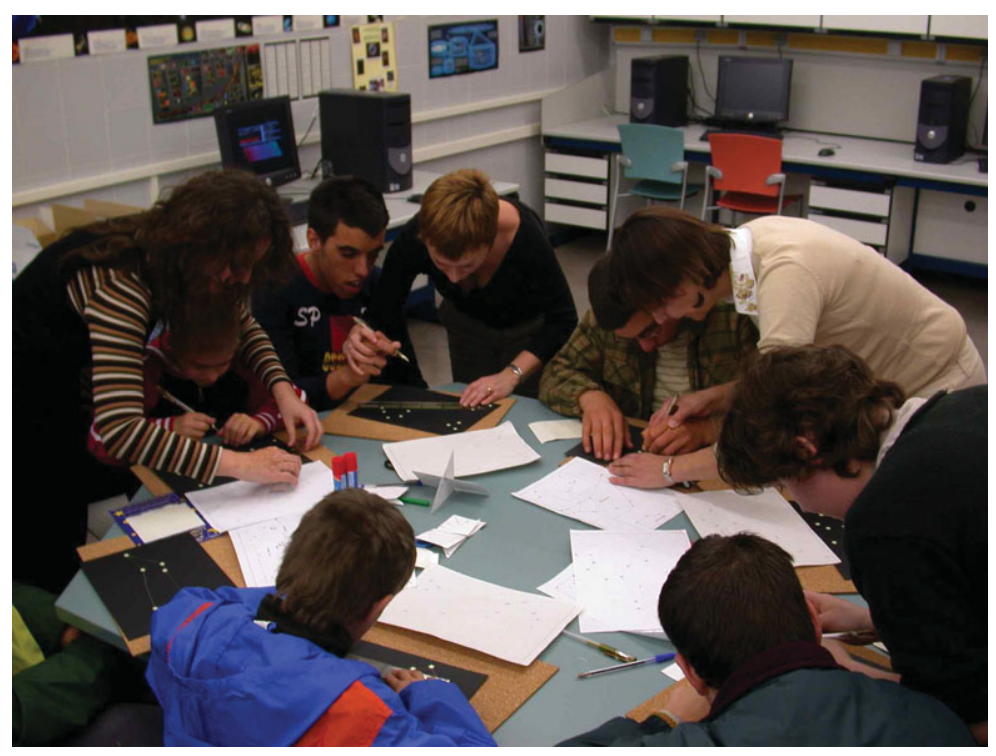

Figure 4. Hands-on Activities: drawing a constellation.

with them. In this case, it is important to know about the intellectual level of the group, and their special needs. With this information in hand, we have organised astronomical workshops for both adults and children groups (Fig. 4).

A short Powerpoint presentation may be a good idea in some cases. We have developed three different sets for these special visits. The first set refers mainly to the physical 
senses, and it is to be watched with sensorial stimula, the public touching cold, warm, soft things according to the presentation. The second one features travelling from the Sun to the Cosmic Microwave Background, with images of the space ships that travelled to the various planets. These two presentations are better shown with some relaxing music. The third presentation is more interactive. Some persons receive a picture which they will have to glue on a large poster when it appears on the screen. The story is about a trip to the Moon and which kind of device one needs to get there. Together with the power point presentations we also offer some hands-on activities. They include building a cardboard sundial, drawing constellations with luminiscent stars or building an astronomical mobile, depending on the abilities of each person.

\section{Where to find these resources?}

All the educational material described here is available (in Spanish) at the Spanish web site for the International Year of Astronomy 2009: http: //www . astronomia2009. es. The web site for the visually impaired, in various languages, is at http://www.touchthesky.eu. Finally, the planetarium recording is available upon request to Amelia Ortiz-Gil amelia.ortiz@uv.es. 